\title{
The Impact of Risk Behaviors on Violence among High School Students
}

\author{
PhD Cand. Ana Uka \\ Department of Educational Sciences, Faculty of Philology and Education, \\ Beder University \\ email: auka@beder.edu.al
}

\section{Doi:10.5901/jesr.2014.v4n2p257}

\begin{abstract}
Current research suggests that the knowledge of the associations between adolescent risk behaviour and delinquent behaviours such as fighting at school or violence among peers is conceptually and empirically inadequate to substantially provide a base of assessment of adolescent health and risk. The aim of this paper is to contribute towards the knowledge and findings about the relationships between adolescent risk behaviours and violent behaviours among adolescents in an educational setting. From a developmental perspective, links between such behaviors in adolescence are discussed and investigated in light of a psychosocial stress model. This report summarizes results from Youth Risk Behavior Survey (YRBS), the 2011 national survey, conducted among students aged 12-18 in grades 9-12. A total of 15,364 students completed the national survey in 2011 where $(N=7656,49.6 \%)$ were male and $(N=7708,50 \%)$ were female students. Bivariate and multivariate logistic regression analyses showed that the odds of fighting at school are increasingly greater as children's frequency of carrying weapon at school $(\beta=1.77, S E=.08)$, playing videogame and watching TV scores separately increase among different races. When all four predictor variables were considered together, they significantly predicted whether or not a student would fight at school, $\left(X^{2}=478.67, d f=5, N=14059, p<.001\right)$. Finally, conclusions for future research, behavioral interventions and educational policies on adolescent health are provided.
\end{abstract}

Keywords: YRBS study, risk behaviors, violence, adolescent

\section{Introduction}

Adolescence is a transition period characterized by a rapid development when young people acquire new knowledge and skills and are faced with many new situations (Boyce et al., 2008; and Hurrelmann \& Richter, 2006). As adolescents go through many physical and emotional changes, they are faced with many pressures and challenges as well, including disadvantages of using technology, the social media, peers using weapons, and academic and behavioral problems. Some risk behaviors such as spending extended hours on watching TV, playing videogame for many hours and carrying weapons or guns at school, established during adolescence can influence the adolescents' social relationships with the others at school. Such adverse behaviours create the roots of a behavioral pattern that might have long-lasting effects at a high cost to the health of young people.

School is such an important social and learning environment influencing not only educational outcomes but also social and behavioral ones. Research shows that children who are not engaged with learning or who have established poor relationships with peers and teachers, are more likely to get involved in socially disruptive and conduct behaviors (Bond, Butler, Thomas, Carlin, Glover, Bowes, \& Patton, 2007). Those children who stay more isolated and more engaged with the social media and technology or those who get involved with behaviors such as holding weapons at school are more likely to develop adverse behaviors that may influence others' health as well. Risk behaviour is considered any behaviour that might have undesirable consequences which increase the probability of harm and loss (Cairns and Cairns, 1994). Although there is a vagueness in the literature about the concepts related to risk behaviour, there is a consensus that such behaviours are directly or indirectly related to health, adverse behaviours and inadequate psychosocial adjustment (Hurrelmann \& Richter, 2006).

Children from different races may show interest on risk-taking behaviors and violent ones at school. Most of the young children, who get involved with the juvenile justice system, usually come from low income single-parent homes found in poor neighborhoods and have high rates of learning problems, mental health issues, and behavior problems. The juvenile justice system reports that one child every 21 seconds is arrested in the US. Children of color ages 10-17 represent only 16 percent of the overall child population ages 10-17, but make up 34 percent of children arrested, 38 percent of children adjudicated, and 68 percent of children in residential placement (Children's Defense Fund, 2014). 
Incarcerated youth are at increased risk of physical abuse, sexual assault and suicide. There are better choices than incarceration that work for children, keep communities safe, and are more cost-effective. Diversion programs, treatment programs, after-school reporting programs, and family support programs help keep children out of trouble.

Adolescents show a tendency to become either more dependent or independent on various factors and peers. Interactions with peers who show deviant behaviors or who are more socially isolated might result in adolescents getting more involved with risk behaviors during this period.

This work is built on the theoretical basis of social control theory (Gottfredson \& Hirschi, 1990). This theory claims that problem behaviors in adolescence are attributed to developed conduct problems early in life. Gottfredson \& Hirschi (1990) characterize delinquents who are predisposed to become as such in childhood as those lacking self-control, or being more impulsive, insensitive and risk-taking. They claim that low self-control can result in criminal behavior only when such an opportunity is present. The lack or the presence of self-control is found in childhood and that is where the problem behaviors first emerge. From a developmental perspective, links between such behaviors in adolescence are discussed and investigated in light of a psychosocial stress model (Cohen \& Wills, 1985). Evidence suggests that psychosocial stress may increase risk for psychosis, especially in the case of cumulative exposure. A heuristically useful framework to study the underlying mechanisms is the concept of "behavioral sensitization" that stipulates that exposure to psychosocial stress-such as life events, childhood trauma, or discriminatory experiences may progressively increase the behavioral and biological response to subsequent exposures (van Winkel, Stefanis \& Germeys, 2008). This study focuses on understanding young people's risk behaviors and their impact on developing problem behaviors such as violence. This work seeks to identify and discuss the extent of these habits or risk behaviors and highlight the need for preventive action to "turn this vulnerable age into an age of opportunity". The aim of this work is to (a) examine the various linkages between risk behaviors and violence in adolescence; and (b) conclude with intervention and policy implications of the findings.

\section{Method}

\subsection{Sampling Procedure and Sample}

The Youth Risk Behavior Surveillance System (YRBSS) is conducted by the Center for Disease Control and Prevention and it monitors six categories of priority health-risk behaviors among youth and young adults: 1 ) behaviors that contribute to unintentional injuries and violence; 2) tobacco use; 3) alcohol and other drug use; 4) sexual behaviors that contribute to unintended pregnancy and sexually transmitted diseases (STDs), including human immunodeficiency virus (HIV) infection; 5) unhealthy dietary behaviors; and 6) physical inactivity. In addition, YRBSS monitors the prevalence of obesity and asthma. YRBSS includes a national school-based Youth Risk Behavior Survey (YRBS) conducted by CDC and state and large urban school district school-based YRBSs conducted by state and local education and health agencies. This report summarizes results from the 2011 national survey, 43 state surveys, and 21 large urban school district surveys conducted among students aged 12-18 in grades 9-12. A total of 15,364 students completed the national survey in 2011 where $(N=7656,49.6 \%)$ were male and $(N=7708,50 \%)$ were female students.

Measures

\subsection{Predictor variables: Carrying weapon.}

The respondents reported their weapon carrying during the past 30 days. Weapon carrying was assessed by asking respondents if they had carried a weapon such as a gun, knife, or club during the past 30 days (from $1=0$ days to $6=6$ or more days), this variable was collapsed into a dummy variable $(1=$ yes, $5.1 \% ; 0=n o, 94.9 \%)$. Watching television. The respondents reported the number of hours they watched television on an average school day (from $1=$ no watching television to $7=5$ or more hours per day), this variable was collapsed into three categories ( $1=$ no television, $27.9 \% ; 2=$ $1-2 \mathrm{hrs} / \mathrm{day}, 36.4 \%$ and $3=3$ or more hrs/day, 35.7\%). Playing videogames. The respondents reported on the number of hours they spent playing video or computer games or used the computer for something that was not school-related (from $1=$ no playing video or computer game to $7=5$ or more hours per day), this variable was collapsed into a dummy variable $(1=$ once or more, $66.8 \% ; 0=$ never, 33.2\%). Race/Ethnicity. The respondents reported their race or ethnicity by selecting one option out of five categories. This variable was collapsed as well into a dummy variable $(1=$ white, $40.8 \%$; $0=$ other, $59.2 \%)$.

Dependent variable. Fighting at school. Fighting was assessed by asking respondents how often they had been in 
a physical fight during the last 12 months (from $1=0$ times to $8=12$ or more times) and this variable was collapsed as well into a dummy variable ( $1=$ once or more, $12.1 \% ; 0=$ never, $86.3 \%)$.

\subsection{Data Analytic Strategy}

We used SPSS 20.0 to analyze the data. Bivariate and multivariate logistic regression analyses were conducted to investigate if there is a relationship between youth risk behaviors and race on violence at school. Firstly, we collapsed both predictor and outcome variables to meet the assumption for the logistic regression analyses which predicts a dichotomous dependent variable when the independent variables are either dichotomous or normal/scale. We also checked for multicollinearity. Because tolerance and VIF scores are not available through the logistic regression command, linear regression analyses were conducted and the results showed that all the variables were approximately .87 , with an adjusted $R^{2}$ of .042 , so there was no multicollinearity.

\section{Results}

Results from the bivariate logistic regression are presented in Table 1 which shows the crude odds ratios (CORs), and 95\% Confidence Intervals (Cls). The results showed that when each predictor variable was entered alone in the model, it significantly predicted whether or not a student fought at school.: 'carrying weapon at school' ( $\beta=1.77, \mathrm{SE}=.08)$, 'watching television (1)' ( $\beta=.07, \mathrm{SE}=.07)$, 'watching television (2)' $(\beta=.25, \mathrm{SE}=.07)$ 'playing videogame' ( $\beta=.11, \mathrm{SE}$ $=.06)$, and 'race/ethnicity' ( $\beta=.45$, SE =.06). Table 1 presents the crude odds ratios for each predictor variable which suggest that the odds of fighting at school are increasingly greater as children's frequency of carrying weapon at school ( $\beta=1.77$, SE =.08), playing videogame and watching TV scores separately increase among different races. Results showed also that youth who watched television for 3 or more hours a day had an increased risk for fighting at school compared to those youth who had not watched television, but there was no increased risk for those who watched television for 1-2 hours/day compared to non-television watchers.

Table 1: Bivariate Logistic Regression Predicting Who Will Fight at School

\begin{tabular}{lcccc} 
Variable & $B$ & SE & CORs & $p$ \\
\hline Carrying weapon & 1.77 & .08 & 5.81 & $<.001$ \\
Watching TV (1) & -.07 & .07 & .68 & $<.001$ \\
Watching TV (2) & .25 & .07 & .74 & $<.001$ \\
Playing videogame & .11 & .06 & 1.19 & 0.001 \\
Race & -.45 & .06 & .62 & $<.001$ \\
\hline
\end{tabular}

Results of multivariate logistic regression analyses shown in Table 2, report that when all four predictor variables were considered together, they significantly predicted whether or not a student would fight at school, $\left(X^{2}=478.67, d f=5, N=\right.$ $14059, p<.001)$. The odds of fighting at school were increasingly greater as children's frequency of carrying weapon at school, playing videogame and watching TV scores together increase while controlling for races.

Table 2: Adjusted Odds Ratios (AORs) and 95\% Confidence Interval (Cls) for Predicting Violence at School -United States, YRBS, 2011

\begin{tabular}{lccc} 
Variable & $\begin{array}{c}\text { Fighting at school } \\
\text { AORs }\end{array}$ & & Cls \\
\hline Carrying weapon & 5,88 & 4,98 & 6,93 \\
Watching TV (1) & 1,07 & .93 & 1,23 \\
Watching TV (2) & 1,28 & 1,12 & 1,47 \\
Playing videogame & 1,11 & .99 & 1,25 \\
Race & 1,57 & 1,44 & 1,78 \\
\hline
\end{tabular}




\section{Discussion}

Data from a nationally representative sample of 12-18 year old boys and girls indicated that fighting at school are increasingly greater as children's frequency of carrying weapon at school, playing videogame and watching TV scores separately and together increase among different races. Evidence gathered over the last two decades shows that disadvantaged social environment and habits are associated with increased behavior risks. Understanding the nature and the function of risk behaviours and their link with violent behaviors helps at developing intervention programs that could provide alternative behaviors which are healthier and fulfill similar needs of adolescents. YRBS study findings show how young people's health changes as they move from childhood into adolescence and their impact on various social and behavioral outcomes. They can be used to monitor young people's behaviors and determine effective behavior improvement interventions.

In fact young people are often neglected as a population group in health statistics, being either aggregated with younger children or with young adults. Little attention has been paid to social environment and habits related to violent behaviors in this group. Adverse behaviours and health experience during this critical period has short and long-term implications for individuals and society. Life-course approaches to behaviour interventions highlight adolescence as critical in determining adult behaviour in relation to issues such as substance use, carrying weapons, playing videogame for extended hours and so on.

This work has been developed to increase knowledge and understanding around violent behaviors in adolescence from the perspective of risk behaviors allowing researchers, policy-makers and practitioners to convene to analyse data, review policies and interventions and formulate lessons learnt. Specific objectives are to document, analyse and increase knowledge and understanding by: (i) translating research on young people's behaviors into policies and action within and beyond the health and education sector; (ii) scaling up intersectoral policies and interventions to promote young people's health; and involving young people in the design, implementation and evaluation of policies and interventions. The findings presented in this study can contribute to different organizations' upcoming strategies for Europe, Health 2020, which is being developed through a participatory process involving Member States and other partners, including the European Union and its institutions, public health associations, networks and civil society. The objective is to ensure an evidence-based and coherent policy framework capable of addressing the present and forecasting future challenges to young people's behaviors.

Young people's behavior choices, including technology dependent habits, engagement with the social media, substance use and weapon use, change during adolescence. Risk behaviors emerge or worsen during this developmental phase and translate into continuing social and behaviour problems

Besides the strengths of this study, there are limitations as well. The data used from the YRBS study included only students who were at school, excluding those who were not in school, we cannot generalize the results to youth who did not attend the school. Another limitation of this study is that the findings do not draw any causality between youth risk behaviors and delinquency, only indicating that as youth displays more risk behaviors they are more likely to be involved with fighting and violent behaviors at school.

Future research is needed to investigate further why some risk behaviors such as carrying weapons at school, watching TV or playing videogame among youth put them at risk for delinquency. These findings have important implications for the timing of behavior interventions and reinforce the idea that investment in young people must be sustained to consolidate the achievements of early childhood interventions. This is vital for individuals as they grow but is also important as a means of maximizing return on programmes focused on investment in the early years and reducing the economic effects of behaviour problems.

\section{References}

Bond, L., Butler, H., Thomas, L., Carlin, J., Glover, S., Bowes, G., \& Patton, G. (2007). Social and School Connectedness in Early Secondary School as Predictors of Late Teenage Substance Use, Mental Health, and Academic Outcomes. Journal of Adolescent Health, 40, 357.e9-357.e18

Boyce, W. F., Davies, D., Gallupe, O. \& Shelley, D. (2008). Adolescent risk taking, neighborhood social capital, and health. Journal of Adolescent Health, 43(3), 246-252.

Cairns, R. B., \& Cairns, B. D. (1994). Lifelines and Risks: Pathways of Youth in Our Time. Cambridge University Press

Children's Defense Fund Report. (2014). The state of America's children.

Cohen, S. \& Wills, T. A. (1985). Stress, social support, and the buffering hypothesis. Psychological Bulletin, 98(2), 310-357.

Gottfredson, M., \& Hirschi, Y. (1990). A general theory of crime. Stanford, CA: Stanford University Press. 
Hurrelmann, K., \& Richter, M. (2006). Risk behavior in adolescence: the relationship between developmental and health problems. Journal of Public Health, 14, 20-28.

Simetin, I., Kern, J., Kuzman, M., \& Pförtner, T. (2013). Inequalities in Croatian pupils' risk behaviors associated to socioeconomic environment at school and area level: A multilevel approach. Social Science \& Medicine, 98, 154-161.

van Winkel, R., Stefanis, N.C., \& Germeys, I.M. (2008). Psychosocial stress and psychosis. A review of the neurobiological mechanisms and evidence for gene-stress interaction. Schizophrenia Bulletin, 34(6), 1095-1105. 